\title{
L'influence des corridors dans le développement régional : le cas de l'East African Community
}

Ronan Porhel et Alain Léon

\section{(2) OpenEdition}

Édition électronique

URL : https://journals.openedition.org/eastafrica/380

DOI : 10.4000/eastafrica.380

ISSN : 2790-1076

Éditeur

IFRA - Institut Français de Recherche en Afrique

Édition imprimée

Date de publication : 1 avril 2014

Pagination : 17-36

ISSN : 2071-7245

\section{Référence électronique}

Ronan Porhel et Alain Léon, «L'influence des corridors dans le développement régional : le cas de l'East African Community ", Les Cahiers d'Afrique de l'Est / The East African Review [En ligne], 48 | 2014, mis en ligne le 07 mai 2019, consulté le 09 décembre 2021. URL : http://journals.openedition.org/ eastafrica/380; DOI : https://doi.org/10.4000/eastafrica.380 


\title{
L'influence des corridors dans le développement régional : le cas de l'East African Community
}

\author{
Ronan Porhel \& Alain Léon
}

\section{Introduction}

Bien que l'EAC suive fidèlement les grandes étapes de l'intégration institutionnelle (de l'union douanière à l'union politique), cette organisation aspire également à développer et à améliorer la coopération sectorielle de la région et ce à l'image la Communauté de développement de l'Afrique Australe (SADC). La volonté de se doter de structures institutionnelles indispensables à la création d'un environnement commun s'accompagne donc incontestablement par la mise en place de projets sectoriels régionaux tels que la gestion de cet espace commun que représente le lac Victoria ou bien la réhabilitation des corridors de transport : le corridor nord de Mombasa à Kampala via Malaba ; le corridor central de Dar es Salaam à Kigoma via Tabora et en Ouganda via Masaka et le corridor sud de Dar es Salaam à Lusaka via Mbeya.

Le commerce des pays de l'EAC avec le reste du monde étant nettement plus important que le commerce inter-régional, la dépendance des États enclavés ${ }^{1}$ vis-à-vis des ports de l'Océan indien, et donc des corridors, est devenue un enjeu crucial. Ces infrastructures de transports permettent par ailleurs de développer la complémentarité des activités au sein du processus d'intégration régionale. Pourtant, malgré l'existence d'importants corridors, force est de constater que ces derniers perdent de plus en plus de leur compétitivité par la hausse régulière des coûts. Le risque est que les entreprises de la région perdent des marchés et ne s'adaptent plus à la concurrence mondiale. Quelles solutions vont alors être apportées par les États membres de l'EAC et quel rôle peut jouer l'institution régionale?

Si le système de corridors est-africain a effectivement participé au développement de la région, il montre aujourd'hui de nombreuses limites (1). Dans une perspective d'avenir, afin de pallier ces difficultés, les États membres prévoient des investissements d'ampleur lesquels seraient plus efficaces avec un approfondissement de l'accord régional (2).

1 Les pays enclavés sont l'Ouganda, le Rwanda, le Burundi mais également les autres pays d'Afrique centrale comme l'Est de la République Démocratique du Congo (RDC) et désormais le Soudan du sud ou l'Éthiopie. 


\section{Les corridors d'Afrique de l'Est : un outil d'intégration régionale indispensable mais qui reste imparfait}

Conformément à l'intégration régionale des espaces, la création d'infrastructures « interconnectantes » a favorisé le dynamisme commercial de la région et l'éclosion de nouvelles activités (1.1.). Mais les perturbations chroniques le long des corridors ainsi que l'incapacité à développer certaines régions révèlent les insuffisances de cet outil (1.2.).

\subsection{La création d'un espace commun basé sur des corridors}

\section{De la nécessité de développer des corridors}

Un corridor de transport est une structure multimodale composée d'axes routiers, ferroviaires, fluviaux ou encore aériens, fournissant alors une connexion économiquement efficiente entre des centres d'activités économiques d'une ou plusieurs zones enclavées d'un ou de plusieurs pays à un pays voisin ayant un accès maritime. Les corridors les plus modernes se composent également des oléoducs, gazoducs, des lignes à haute tension ou de la fibre optique. Aussi, la région de l'EAC connait un système de corridors développé depuis la période coloniale britannique.

Dans son processus de développement, l'EAC a intégré une union douanière en 2005 puis un marché commun en 2010. Mais l'élaboration de cette communauté économique régionale (CER) s'inscrit également dans l'intégration d'un espace commun lequel produit un double effet d'agglomération et de diffusion tel qu'évoqué par Paul Krugman dans la nouvelle économie géographique ${ }^{2}$. Aussi une région défavorisée, désirant être reliée à un processus de développement en cours, doit créer les conditions de son attractivité en s'attachant à instaurer un "capital public spatialisé $»^{3}$. Compte tenu des forts coûts de transports dans les pays en développement (PED), en particulier à destination des pays enclavés ${ }^{4}$, les principales institutions internationales, dont la Banque Mondiale, ont reconnu l'impact bénéfique des investissements en infrastructures "interconnectantes » entre différents territoires sur la croissance des pays africains ${ }^{5}$.

\footnotetext{
2 La nouvelle économie géographique montre le rôle décisif de l'espace dans l'émergence d'économies d'agglomération en raison de la concentration de producteurs d'une même branche. À ce titre elle rejoignait les conclusions de G. Myrdal sur le concept de croissance déséquilibrée par une phase de concentration puis de diffusion. Voir Krugman, P., «Increasing returns and economic geography », Journal of Political Economy, nº99, Vol. 31, 1991, pp. 483-499.

3 Le capital public spatialisé peut être représenté par des services publics comme les transports, les télécommunications, la recherche etc. Voir Akanni-Honvo, A. \& Léon, A., « La croissance endogène régionalisée ", Revue Tiers Monde, $\mathrm{n}^{\circ} 155$, juillet-septembre, 1998 , Paris.

4 Selon la Banque Mondiale, les coûts de transport dans les pays enclavés peuvent atteindre $77 \%$ de la valeur des biens exportés. Avant d'être à bord d'un navire, un conteneur coûte en moyenne 872 Dollars US (USD), soit $703 €$ en transport, contre 300 USD en Chine.

5 Selon une étude de la Banque Mondiale, un taux de croissance annuel de $13 \%$ des infrastructures est corrélé à 1 point de croissance économique par tête. Voir « World Bank, The Africa Competitiveness », World Economic Forum Report, 2013.
} 
Dès la période 2001-2005, la stratégie de l'EAC, ayant pour but de faire participer l'ensemble des territoires à la dynamique régionale, a donc été de tisser un maillage d'infrastructures du local au régional. Ces interconnexions doivent permettre de créer un grand marché susceptible d'exploiter toutes les potentialités de la zone en terme de complémentarité des structures productives (Kenya : place boursière et main d'œuvre qualifiée ; Tanzanie : ressources naturelles ; Ouganda : hydroélectricité, etc.). Dans ce contexte, les États partenaires espèrent attirer les investisseurs étrangers pour financer de nouvelles activités.

\section{L'espace est-africain s'appuie sur trois corridors majeurs :}

L'espace est-africain se structure autour de deux axes principaux, le corridor nord (de Mombasa à Kampala via Malaba) et le corridor sud (Dar es Salaam à Lusaka via Mbeya), intercalé par un troisième couloir commercial le corridor central (Dar es Salaam à Kigoma via Tabora et en Ouganda via Masaka) ${ }^{6}$. Les dynamiques régionales étant depuis longtemps tournées vers l'exportation de matières premières, l'essentiel des richesses se produisent le long de ces corridors et l'entité est-africaine s'est créée à partir de cet $\mathrm{ADN}^{7}$.La réactivation de ces trois couloirs commerciaux a vite constitué une priorité pour rendre ces espaces plus communicants et réduire des coûts de transaction très élevés.

Le corridor nord, de loin le plus fréquenté, traverse l'intérieur du Kenya de Mombasa à Malaba via Nairobi. Après le poste frontière de Malaba, le corridor s'éclate en deux segments, l'un prenant la direction de Kampala (Ouganda), du Rwanda et de la République Démocratique du Congo et l'autre allant vers le Sud Soudan et l'Éthiopie. Point de transit incontournable, aussi bien pour les importations que pour les exportations ${ }^{8}$, le port de Mombasa est raccordé aux principales villes de l'hinterland, Kampala en Ouganda, Kigali au Rwanda, Bujumbura au Burundi ainsi qu'à Goma et Kisangani en République Démocratique du Congo (RDC). Entre 2007 et 2009, l'activité du port de Mombasa a augmenté de $40 \%$, ( $+30 \%$ pour les containers) en raison du dynamisme des pays enclavés et de l'apparition du Sud-Soudan comme nouveau partenaire. Les 1898 kilomètres du corridor nord sont en très grande partie bitumés sur une seule voie 9 . Par ailleurs, une concession importante du réseau ferré de Kenya Railway et Uganda Railway a été accordée à un exploitant privé sud-africain, la Rift Valley Railway (RVR) en 2005. Cette concession est cependant effective seulement depuis 2010. En raison de défauts d'entretien et de pénuries d'équipements, seul $5 \%$ du trafic de ce corridor utilise ce mode de transport. Un oléoduc jusqu'à la ville d'Eldoret et un système de voies navigables sur le lac Victoria viennent compléter la structure. Un

\footnotetext{
6 Voir carte de l'EAC et ses corridors en annexe 2.

7 Selon Hervé Maupeu, politiste et spécialiste de l'Afrique orientale à l'Université de Pau et des Pays de l'Adour. $895 \%$ des marchandises qui sont importées vers - ou exportées de - l'Ouganda transitent par le port de Mombasa. Voir Trade Facilitation in the East African Community: Recent Developments and potential Benefits, US International Trade Commission. Publication n ${ }^{\circ} 4335$. July 2012.

9 Selon une étude menée, en 2010, par la firme Aurecon Engineering pour le East African Transport Strategy and Regional Road Sector Development Program, les trois quarts du réseau routier du corridor nord sont notés «acceptables » à « bons »; mise à part le segment reliant le Rwanda et le Burundi.
} 
début d'allègement des procédures aux trois postes frontières ${ }^{10}$, ainsi que la modernisation des installations, ont permis de diviser par deux environ les distances économiques entre 2005 et $2011^{11}$. Pour certains chefs d'entreprise comme Robert Byaruhanga, responsable de la logistique au sein de l'entreprise ougandaise Kyagalanyi Coffee Ltd., les améliorations du corridor nord ont d'ores et déjà eu des retombées bénéfiques et permettent de réaliser des économies sur les frais de transit : "Cela prenait tellement de temps avant, il fallait en moyenne compter 40 à 50 jours pour faire expédier un chargement de café de Kampala jusqu'à un bateau. Mais, aujourd'hui, nous nous en sortons en 18-23 jours ».

Le corridor sud, composé d'un réseau routier, ferroviaire sur l'ensemble de la distance et d'un oléoduc jusqu'à Ndola, raccorde le port de Dar es Salaam en Tanzanie à Lusaka en Zambie via Mbeya. L'écartement austral (1,6 mètres) de la voie ferrée de la compagnie Tazara permet de relier les pays membres de la Communauté de développement d'Afrique Australe (SADC) au port tanzanien.

Le corridor central représente le principal hub d'échanges pour la Tanzanie et les deux derniers adhérents de l'EAC, le Burundi et le Rwanda ${ }^{12}$ mais reste, pour le moment, marginal pour l'Ouganda en raison d'un coût peu compétitif. Il relie Dar es Salaam à Kigoma via Tabora et fait la jonction avec l'Ouganda via Masaka par la route ou par voie maritime à travers le lac Victoria entre Mwanza et Port Bell. La voie ferroviaire s'arrêtant à Mwanza, la liaison se fait essentiellement par un réseau routier désormais bitumé à $86 \%{ }^{13}$. Par contre, comme son homologue du nord, le corridor est constitué d'une seule voie sur $90 \%$ de leurs parcours respectifs ${ }^{14}$. Entre 2006 et 2009, l'activité du port de Dar es Salaam, pourtant moitié moins importante que celui de Mombasa, s'est accrue de $48 \%$ ( $+22 \%$ pour les containers) ${ }^{15}$.

10 Les trois postes frontières du corridor nord se situent à Malaba (Kenya-Uganda), Gatuna-Katuna (OugandaRwanda), and Akyaru-Kinyaru Haut (Rwanda-Burundi). Entre 2005 et 2011, mise à part le cas du Burundi les documents à fournir pour les importateurs et les exportateurs ont nettement baissé : 4 pour le Kenya, 18 pour le Rwanda, 9 pour la Tanzanie et 15 pour l'Ouganda. En 2011, le nombre de documents exigé pour les importateurs, par exemple, s'élève à 6 pour la Tanzanie et 10 pour le Burundi. Voir Trade Facilitation in the East African Community: Recent Developments and Potential Benefits, op. cit.

11 Selon le «Trade Mark East Africa » (TMEA), institution responsable de l'observation des principaux corridors de la région sous l'égide du programme des politiques de transport en Afrique Sub-saharienne (SSATP), entre 2005 et 2011, en moyenne, les délais de passage de la frontière à Malaba ont été réduits de plus de 3 jours à 3-5 heures. Le temps d'immobilisation au port de Mombasa est réduit de 19 à 8 jours et le temps de transit le long du tronçon Mombasa-Nairobi-Kampala a baissé de 15 à 5 jours.

$1257 \%$ des importations et $65 \%$ des exportions du Rwanda passent par le corridor central. Les chiffres sont respectivement $94 \%$ et $98 \%$ pour le Burundi. Voir Trade Facilitation in the East African Community..., op. cit.

13 Étude menée, en 2010, par la firme Aurecon Engineering pour le East African Transport Strategy and Regional Road Sector Development Program.

14 Trade Facilitation in the East African Community ..., op. cit.

15 Entre 2007 et 2009, les volumes traités au port de Mombasa sont passés de 10,7 à 15 millions de tonnes dont 30\% de containers. Entre 2006 et 2009 les volumes échangés au port de Dar es Salaam sont passés de 7,2 à 10,6 millions de tonnes. Voir Society for International development (SID), The State of East Africa 2012. Deepening Integration, Intensifying Challenges. Nairobi, SID, 2012. 
Ce système structuré entre le hub aéroportuaire de Nairobi, les deux grands ports de l'Océan indien (Mombasa et Dar es Salaam) et des villes intermédiaires servant de lieux de liaison, a amené les acteurs à considérer cette zone comme une seule et même unité tarifaire anticipant dès lors la vision régionale de $1^{\prime} \mathrm{EAC}{ }^{16}$. Si des voies de communication sommaires restent encore assez fournies entre ces trois principaux segments, les versants nord et sud de l'Afrique Orientale se particularisent cependant par l'extrême pauvreté de l'infrastructure. En concentrant ses dépenses sur les zones à fort potentiel, situées autour de Nairobi, les autorités kenyanes ont délaissé les axes nord $/ \mathrm{sud}^{17} \mathrm{mise}$ à part la portion de route Arusha-Nairobi via Namanga. Le quadrillage de la région est donc loin d'être atteint. Seul $9 \%$ du réseau de l'EAC est bitumé.

\section{Un commerce en hausse grâce à l'apport des corridors}

La réhabilitation de liaisons routières infra-nationales et régionales d'une part, et la mise en œuvre de l'union douanière d'autre part, se sont traduites par des effets d'agglomération autour des points de connexion et des créations de commerce. Le commerce entre les pays de 1'EAC a presque doublé entre 2005 et 2010 en passant de 2,2 à 4,1 milliards USD. Le commerce régional avec le reste du monde a augmenté encore plus vite, signe de la vitalité des corridors de transport.

\section{Les exportations de l'EAC et le commerce intra-régional}

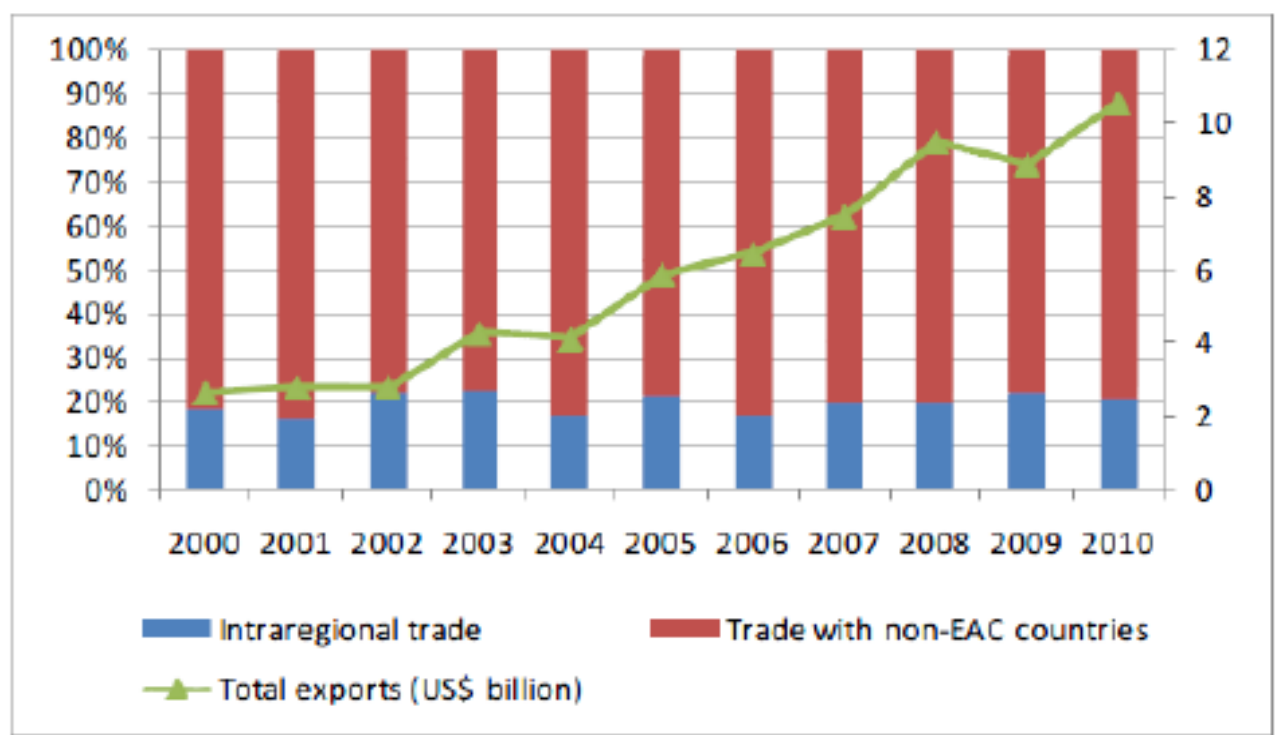

Source: United Nations Comtrade via World Integrated Trade Solutions

16 Calas, B. « Le système logistico-urbain est africain », Mambo!, IFRA, 2000.

17 En l'absence de connexions de qualité, les surplus alimentaires du sud et de l'ouest de la Tanzanie peuvent difficilement combler les déficits des régions nord du Kenya, de l'Ouganda mais également de la Somalie et de l’Éthiopie. 
Ce développement du commerce s'est accompagné d'une hausse des débouchés et d'une diversification des exportations grâce au dynamisme de nouvelles activités. Même si les productions périssables à forte valeur ajoutée sont plutôt utilisatrices du transport aérien, celles-ci ont également besoin de s'appuyer sur un réseau routier performant. C'est le cas des secteurs de la pêche et surtout de l'horticulture. À la différence des pêcheries tanzaniennes et ougandaises qui empruntent les aéroports de proximité de Mwanza et d'Entebbe, celles localisées autour de Kisumu doivent exporter leurs filets de perche, à partir de l'aéroport de Nairobi, à plus de 300 kilomètres. La modernisation et le prolongement du corridor nord ont donc permis de respecter davantage les délais à l'export et d'élargir le marché local et régional, pour les « bas morceaux », jusqu'au cœur de l'Afrique centrale et au Sud Soudan.

L'horticulture est un secteur prometteur pour ses perspectives de croissance et les dotations naturelles dont sont pourvues les pays de la zone ${ }^{18}$. L'essentiel des cultures horticoles, incluant les fruits, les légumes et les fleurs coupées, sont concentrées dans la région du lac Naivasha au Kenya, autour d'Arusha et de Moshi en Tanzanie. Mais du fait de leur extrême fragilité, elles doivent avoir atteint leur point de vente, local ou international, dans les 24 heures suivant la cueillette. La modernisation du réseau routier du corridor nord et de la liaison Arusha Nairobi via Namanga étaient des conditions pour que ces produits à forte valeur ajoutée puissent être exportés au plus vite à partir de l'aéroport de Nairobi. Selon le « Kenya Flower Council », le secteur a rapporté en 2011, 1,087 milliards USD devenant le plus important générateur de devises. Lors des quatre années précédant la crise économique mondiale, le secteur horticole en Tanzanie a connu une croissance de $8 \%$ à $10 \%$ par an.

\subsection{Un système de corridors toujours faillible}

Si le corridor nord a permis la concentration d'activités dans un rayon de 100 kilomètres autour de la ligne ferroviaire reliant le Kenya à l'Ouganda ${ }^{19}$, il a également accentué la marginalisation économique des communautés vivant dans les régions arides du nord et les zones rurales et a échoué à rendre attractives les zones portuaires de 1'Océan indien. Ainsi, malgré le constat d'une hausse du revenu par habitant dans la région, le nombre de personnes vivant sous le seuil de pauvreté, moins de 1,25 USD par jour selon la Banque mondiale, a augmenté de 44 à 53 millions de personnes entre 1994 et 2010 pour atteindre $38 \%$ de la population de la région ${ }^{20}$. Les trois comtés de l'extrême nord du pays sont, de loin, les plus pauvres du pays. 94,3\% des habitants du Turkana vivent sous le seuil de pauvreté, contre 22\% dans la capitale Nairobi, selon le dernier recensement de 2009.

18 Situé aux abords de l'équateur, le secteur horticole de ces pays peut fonctionner toute l'année.

19 Déclaration de M. Cyrus Njiru, Secrétaire Permanent de l’EAC aux Transports en juin 2012. www.

theafricareport.com

20 Society for International Development (SID), The State of East Africa 2012..., op.cit. 
Par ailleurs, malgré les améliorations enregistrées depuis les années 2000, les États membres ont obtenu de faibles indices de performances logistiques ${ }^{21}$ dus notamment au retard de compétitivité des transports régionaux ${ }^{22}$. Comparé aux USA et à l'Europe, le surcoût par kilomètre s'élève encore à $50 \%$ pour les deux corridors et même à $75 \%$ pour les pays enclavés ${ }^{23}$. Une étude du Conseil kenyan des transporteurs (KSC) a révélé qu'un conteneur de 40 pieds nécessite 28 jours de Shanghai (Chine) à Mombasa et coûte 6000 USD, alors que le même conteneur nécessite 41 jours de Mombasa à Bujumbura pour 8000 USD.

Indices de performance logistique dans l'EAC (cf. paragraphe précédent)

\begin{tabular}{|c|c|c|c|c|c|c|c|}
\hline & \multicolumn{5}{|c|}{ EAC } & \multirow{2}{*}{$\begin{array}{r}\text { Toplow- } \\
\text { income } \\
\text { country }\end{array}$} & \multirow{2}{*}{$\begin{array}{r}\text { Bes } \\
\text { practices } \\
\text { countr } \\
\text { Singapo'e }\end{array}$} \\
\hline & Burundi & Kenya & Rwanda & Tanzania & Uganda ${ }^{a}$ & & \\
\hline \multicolumn{8}{|l|}{$\begin{array}{l}\text { Logistics performance index scores } \\
\text { on a scale from } 1 \text { (wcrst) to } 5 \text { (best) }\end{array}$} \\
\hline Aggregate score & 1.61 & 2.43 & 2.27 & 2.65 & $2.82^{*}$ & 285 & 4.13 \\
\hline Efficiency of customs procedures & 1.67 & 2.08 & 2.19 & 2.17 & $2.84^{*}$ & 259 & 4.10 \\
\hline Percentage of cargo inspected & $60 \%$ & $25 \%$ & 19 & (9) & 7596 & $11 \%$ & $1 \%$ \\
\hline Infrastructure quality & 1.68 & 2.16 & 1.88 & 2.41 & $2.35^{*}$ & 257 & 4.15 \\
\hline Ease of shipping internationally & 1.57 & 2.69 & 2.27 & 2.91 & $3.02^{*}$ & 2.44 & 3.99 \\
\hline Timeliness of deliveries & 1.67 & 2.88 & 2.76 & 2.97 & $3.52^{*}$ & 3.74 & 4.39 \\
\hline \multicolumn{8}{|l|}{ Trading across borders indicators } \\
\hline Documents needed to import & 10 & 7 & 8 & 6 & 9 & 8 & 4 \\
\hline Documents needed to export & 9 & 3 & 8 & 6 & 7 & 7 & 4 \\
\hline Days needed to import & 54 & 24 & 31 & 24 & 34 & 32 & 4 \\
\hline Days needed to export & 35 & 26 & 29 & 18 & 37 & 30 & 5 \\
\hline
\end{tabular}

Source. World Bank, "Logistics Performance Index," 2010, 2012; World Bark, "Doing Business," 2012.

"Starred "Logistics Performance" data for Uganda are from the 2010 report. All other data are from the 2012 version.

bBenin was the highest-ranked low-ncome country in the 2012 Logistics Performance Index. It was ranked ninth among low income ccuntries for Doing Business' Trading Across Borders rankings in 2012.

'Not available

21 Sur une échelle de 1 à 5, les indices de performance logistique des pays de l'EAC sont de 1,61 pour le Burundi à 2,82 pour l'Ouganda, en deçà de la moyenne des pays de l'Afrique sub-saharienne. Construit par la Banque Mondiale, l'indice de performance de la logistique reflète les perceptions relatives à la logistique d'un pays basées sur l'efficacité des processus de dédouanement, la qualité des infrastructures commerciales et des infrastructures de transports connexes, la facilité de l'organisation des expéditions à des prix concurrentiels, la qualité des services d'infrastructure, la capacité de suivi et de traçabilité des consignations et la fréquence avec laquelle les expéditions arrivent au destinataire dans les délais prévus.

22 Les coûts de transport depuis le port de Mombasa : 0,04 USD/km pour le Kenya ; 0,085 USD pour l'Ouganda ; 0,09 USD pour le Rwanda et 0,11 USD pour Burundi. Voir Transport Prices and Costs in Africa; BIRD, World Bank, 2009.

23 Trade Facilitation in the East African Community..., op. cit. 
Ce problème de compétitivité des transports est lié notamment aux dysfonctionnements des deux portes océanes de l'EAC (Mombasa et Dar es Salaam) ainsi qu'à la présence de barrières non tarifaires, le long des corridors. Ces éléments sont en effet en grande partie responsables du ralentissement du trafic et de l'incertitude relative au commerce en général.

Concernant le dysfonctionnement portuaire (des deux portes océanes), le port de Mombasa, conçu à l'origine pour accueillir 250000 conteneurs (EVP), en a traité, en 2012, plus de $800000^{24}$. Cet engorgement contraint les bateaux à stationner avant d'accoster, près de 15 jours, à un coût journalier entre 10000 et 12000 USD, Mais pour Wolfgang Fengler, chef économiste de la Banque Mondiale au Kenya, le principal problème provient surtout de l'inefficacité des installations portuaires ${ }^{25}$. À Mombasa, une multitude d'organisations ${ }^{26}$ présentes sur le port et dont on ne connait pas toujours les véritables responsabilités, ralentissent non seulement le traitement des containers mais favorisent également les tentations de corruption ${ }^{27}$. Le site se trouve par conséquent rapidement saturé d'autant plus que certains importateurs utilisent le port comme lieu de stockage. Cette situation toucherait d'ailleurs davantage les produits des pays enclavés. $75 \%$ des importations ougandaises sont inspectées, contre $25 \%$ pour celles à destination du Kenya, alors que les produits ont, pour la plupart d'entre eux, déjà été contrôlés par des laboratoires accrédités ${ }^{28}$. Outre cette impression de discrimination ${ }^{29}$, ces pays sans littoral subissent déjà une combinaison interactive de facteurs très variés ${ }^{30}$ qui contraignent le positionnement de leurs productions sur les marchés mondiaux et surtaxent les produits importés. La Banque Mondiale estime que le temps de dédouanement dans ce port est sept fois moins prévisible que dans les autres régions. Cette inefficacité nécessite $50 \%$ à $80 \%$ de temps en plus pour les importateurs des pays enclavés.

A la sortie du port, les corridors routiers sont largement plus utilisés ${ }^{31}$ que le réseau ferroviaire pourtant plus économique à la tonne-kilomètre $(0,05$ USD par kilomètre pour une tonne contre 0,09 USD par la route). Mais la mauvaise qualité du réseau le rend assurément plus imprévisible.

24 «Congestion at Mombasa port slows down trade in EAC bloc », The East African, 13 décember 2012.

25 Selon Jacques Morisset, chef économiste de la Banque Mondiale en Tanzanie, l'inefficacité du port de Dar es Salaam pourrait être à l'origine d'une perte de 1,8 milliards USD pour le pays soit $7 \%$ du PIB. Voir «Inefficiency at Dar port swallows up \$2,6 bn of GDP», The East African, 25 mai 2013.

26 The Kenya Ports Authority (KPA), Kenya Revenue Authority (KRA), Kenya Bureau of Standards, Kenya Plant Health Inspectorate Services, Port Health Authority (PHA), Dairy Board of Kenya, National Bio-Safety Authority, Anti-Counterfeit Agency and Port Police.

27 Plusieurs scandales durant le régime Kibaki ont montré que ces filières de corruption à partir du port de Mombasa sont toujours au cœur du système clientéliste des élites kenyanes.

28 Trade Facilitation in the East African Community: Recent Developments and potential Benefits, op. cit.

29 World Bank, East African Community. Reshaping Economic Geography of East Africa: From Regional to Global Integration, Report nº5699-AFR, June 2012.

30 Ces facteurs sont : la géographie physique (distances ; climat ; densité végétale, systèmes orographiques) ; les performances logistiques (état des infrastructures, traçabilité) ; les problèmes politiques et humains (gouvernance, corruption, barrages illégaux, instabilité politique et ethnique) et enfin le cadre réglementaire. Voir Alix, Y. \& Pelletier, J.-F., «Territoires enclavées et opportunités de marché : analyse des performances logistiques des corridors de transport en Afrique subsaharienne », Organisations et territoires, 2011.

31 Selon Polycarp Igathe, chairman of the Kenya Association of Manufacturers : "le port de Mombasa est peut-être le seul où $95 \%$ des bateaux sont évacués par la route. C'est totalement insoutenable". 
Cependant, sur les routes, un problème de taille se pose depuis plusieurs années : les nombreuses barrières non tarifaires. Elles allongent considérablement la durée du trajet entrainant des coûts annexes ainsi qu'une baisse de compétitivité des entreprises. Ces barrières peuvent revêtir différentes formes, tel que le manque d'harmonisation des procédures aux différents postes frontières, tel aussi la mise en place de " ponts à bascules " pour préserver l'infrastructure routière en stoppant à chaque arrêt les camions pendant près de trois heures ${ }^{32}$, ou encore le nombre notable de barrages routiers souvent informels ${ }^{33}$. Malgré la volonté ces dernières années de réduire ces barrières non tarifaires, notamment par l'instauration du système informatique de partage de fichiers douaniers Radex et d'opérations de gestion aux postes frontières (OSBP), le corridor nord continue pourtant de les développer. En 2013, 26 jours sont en moyenne nécessaires pour importer un container en Ouganda contre 24 jours l'année précédente. Par cette dégradation du commerce transfrontalier, le World Bank's Ease of Doing Business survey a abaissé le rang

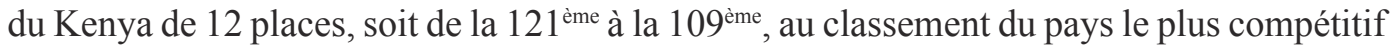
au monde ${ }^{34}$.

Particulièrement sensible à l'imprévisibilité de la durée des transports, le secteur du textile, confronté à la congestion des deux ports de l'Océan indien est contraint d'anticiper et donc d'accroître ses stocks de tissus et de divers inputs pour ne pas se retrouver en rupture de $\operatorname{stock}^{35}$. Les coûts additionnels et l'impossibilité de respecter régulièrement les délais de livraison empêchent ce secteur d'attirer les grandes firmes mondiales de l'habillement.

Cette incertitude et ces surcoûts, portés à leur paroxysme lors des violences postélectorales de 2007/8 $8^{36}$, ont d'ailleurs incité certains professionnels de l'hinterland à se tourner vers le corridor central. Le port de Mombasa a ainsi perdu, en 2011, 18,9 \% de cargaisons provenant de la Tanzanie et des autres partenaires de l'EAC au profit de celui de Dar es Salaam ${ }^{37}$. Mais la capitale économique de la Tanzanie pourrait également être menacée face à l'offensive des ports d'Afrique Australe. Sans une solution appropriée, des pays comme la Zambie, le Malawi ou la région du Katanga (est-RDC) pourraient se diriger vers le Port de Beira (Mozambique) où les prix sont plus abordables et les services efficaces.

Parallèlement à ces limites, les corridors sont également inadaptés aux nouveaux enjeux de la région. En effet la structure logistique actuelle des corridors peine à supporter une croissance du trafic de $23 \%$ entre 2007 et 2011. À ceci s'ajoute l'apparition de nouveaux partenaires, dont le Sud-Soudan, ainsi que le dynamisme des pays enclavés, notamment

32 Les pertes journalières, en raison de la contrainte des ponts à bascule, s'élèveraient à 1200 dollars par camion. Voir "The real cost of trouble at the weighbridge ", The East African Paper, 2013. 33 The 2011 Business Climate Index Survey estime à 24 le nombre de barrages routiers au Kenya et 21 en Ouganda.

34 "Kenya grants Uganda licence for Mombasa freight station ", The East African, 20 mai 2013. 35 Selon une étude de la Banque Mondiale, avec davantage de prévisibilité, les importateurs pourraient mieux gérer leurs stocks en les abaissant de 45 à $50 \%$ d'où une économie de coûts.

36 Porhel, R. «Les conséquences économiques de la crise politique », Les élections générales de 2007 an Kenya, Nairoib/ Paris, IFRA/ Karthala, 2008.

37 Africa Research Bulletin, janvier 2012. 
suite à la découverte de gisements pétroliers en Ouganda, dans les provinces orientales de la $\mathrm{RDC}$ et plus récemment autour du lac Turkana, lesquels vont contribuer dans les prochaines années à un développement des échanges de l'ordre de $8 \%$ par an. L'acheminement de ces produits pétroliers ainsi que l'électricité des barrages et éventuellement le gaz du sud-ouest de la Tanzanie vient relancer le débat sur les modes de transport.

En tout état de cause, les corridors d'Afrique de l'Est soulèvent de nombreux problèmes auxquels les États membres doivent répondre dans les meilleurs délais afin d'améliorer la compétitivité globale de la zone.

\section{Quelles perspectives?}

Face à cette situation, les autorités kenyanes et tanzaniennes ont décidé de mettre l'accent sur une modernisation de la structure logistique. L'amélioration des infrastructures régionales constitue un préalable indispensable à la diffusion de la croissance à l'ensemble des États membres et à l'interconnexion des villes motrices avec les zones marginales. Ainsi les investissements sur le territoire kenyan se sont portés sur la consolidation du corridor nord doublé par la construction d'une nouvelle voie multimodale partant de Lamu (2.1) mais les craintes des pays enclavés, vis-à-vis du pouvoir kenyan, les ont conduits à vouloir accélérer la modernisation des axes tanzaniens (2.2). Toutefois, si ces projets peuvent ajuster la structure logistique aux ambitions de la région, l'EAC ne pourra faire l'économie d'un approfondissement de sa stratégie d'intégration (2.3).

\subsection{Les projets kenyans confortent sa place de leader logistique}

L'objectif est double. D'une part, permettre au Kenya d'affirmer sa position

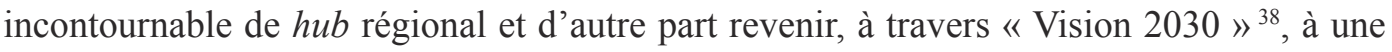
intégration par le haut. C'est-à-dire permettre aux instances de créer, à l'aide d'infrastructures, de la croissance économique et ce dans un contexte d'intense néo-libéralisme.

Dans la lignée de la rencontre tripartite entre les présidents ougandais, kenyan et rwandais à Entebbe en juin 2013, la modernisation du corridor nord doit se penser au regard de la filière pétrole que l'Ouganda souhaite développer. Ainsi, le vieux projet d'extension de l'oléoduc d'Eldoret vers Kigali via Kampala a été de nouveau étudié et ce plus de cinq ans après avoir été attribué à la société libyenne Tamoil, avec l'espoir enfin qu'il se réalise ${ }^{39}$. La construction d'une raffinerie à l'ouest de l'Ouganda est également en projet en raison des faibles capacités de celle de Mombasa. Le désengorgement du corridor nord implique un agrandissement du port de Mombasa à travers la construction d'un second terminal et par

38 «Vision $2030 »$ est un processus de développement étalé sur la période 2008-2030, à l'initiative de l'ancien président kenyan M. Kibaki, devant faire passer le Kenya au statut de pays à revenu intermédiaire. Il s'appuie sur trois piliers économique, social et politique. Il s'agit de transformer l'économie du continent en s'appuyant sur le commerce maritime et le désenclavement des pays de l'Est.

39 «Bilateral and Tripartite Deals: presidents agree to fast track political federation », The East African, 29 juin/5 juillet 2013. 
un projet de modernisation du réseau ferroviaire. Les capacités augmenteraient ainsi à 1,2 million containers par an. La construction de trois nouveaux postes de mouillage permettra d'accueillir la dernière génération de porte-container capable de transporter 6000 unités contre 2400 pour les précédents. De même, un projet de construction d'une nouvelle ligne de chemin de fer reliant Mombasa à Nairobi est en voie de réalisation avec l'entreprise chinoise « China Roads and Bridges Company ». Cette voie ferroviaire a pour finalité de se connecter avec l'Ouganda puis se scinderait en deux segments l'un vers le Rwanda et le Burundi l'autre vers Juba au Soudan du Sud ${ }^{40}$. D'une vitesse de 80 kilomètre/heure pour les cargaisons, la durée de transit devrait s'abaisser et encourager les transporteurs à éviter un réseau routier saturé ${ }^{41}$. Enfin, le secrétariat de l'Autorité de coordination du transit du Corridor Nord (ACTT-CN) a poussé les participants à recommander au Burundi d'accélérer les travaux d'élargissement des routes de Kanyaru Haut-Bujumbura et Bujumbura-Gatumba, pour répondre aux normes de l'EAC pour les routes régionales. À long terme, le Burundi et la RDC sont appelés à construire un pont à deux voies à Gatumba/Kavimvira pour accueillir également le poids brut maximal des véhicules de 56 tonnes accepté sous la tripartite (EACCOMESA/SADC). Suite à ces travaux, est attendu une baisse de 40,25 USD par tonne ( $27 \%$ ) pour le corridor nord et par la suite une hausse de $15 \%$ du commerce ${ }^{42}$.

Mais le besoin d'itinéraires alternatifs pour les produits pétroliers du Sud-Soudan et de l'Éthiopie ${ }^{43}$, ainsi que la découverte de gisement de pétrole dans la région du Turkana, ont incité les autorités kenyanes à réveiller un vieux projet d'axe de transport multimodal des années 70. Projet majeur de la « Vision 2030 » du Kenya, officiellement lancé le 2 mars 2012, conjointement entre le Sud-Soudan, le Kenya et l'Ethiopie, le «Lamu-Southern Sudan-Ethiopia Transport» (LAPSSET) a commencé à se concrétiser véritablement avec la construction de 3 des 32 mouillages à Manda Bayde (Lamu). Il s'agit aussi de le relier par un double réseau ferré et routier à Juba et Addis-Abeba via Lokichoggio et Moyale. D'après les experts, le chemin de fer devrait assurer $96 \%$ du chargement à destination du Sud-Soudan et $93 \%$ du chargement à destination de l'Éthiopie. En outre, l'oléoduc, devant permettre au tout jeune Sud-Soudan d'exporter son pétrole à partir de 2030, sera complété par la constitution d'une raffinerie de pétrole sur site portuaire capable de traiter 120000 barils/jour. Ces infrastructures nouvelles et plus denses, qui reposent sur des liaisons à plusieurs couches, pourraient, à l'avenir, favoriser une concentration de nouvelles activités, dans les régions jusqu'ici isolées, le long des corridors, et autour des ports à l'image du corridor de Maputo au Mozambique ${ }^{44}$.

40 "Kenya and China secretly strike deal for new railway", The East African, September 22-28, 2012

41 Avec la modernisation de son réseau ferroviaire, le corridor nord pourrait devenir l'itinéraire privilégié du Rwanda. «Bilateral and Tripartite Deals...», op.cit.

42 Trade Facilitation in the East African Community..., op. cit.

43 L'Éthiopie, qui a recourt au port maritime de Djibouti, est à la recherche de routes plus courtes vers la côte depuis que du pétrole a été découvert dans la région d'Ogaden, frontalière avec la Somalie. Le Sud-Soudan, fraîchement indépendant, est à la recherche d'un itinéraire alternatif pour transporter le pétrole, à cause des conflits dans lesquels il est engagé avec son voisin du nord, Khartoum.

44 Blanc, M.O., Stratégie des acteurs, dynamiques urbaines et structuration des territoires. Sous projet Afrique Australe, Paris, PRUD, projet $\mathrm{n}^{\circ} 37$, janvier 2004. 
Cependant ce scénario le plus probable comporte certaines limites. Tout d'abord, ce projet aura pour challenge de surmonter des problèmes sécuritaires. En effet, le port de Lamu s'ouvrira sur une zone maritime dans laquelle les pirates sont très actifs et à proximité de la Somalie où, malgré l'action de la mission de l'Union africaine (Amisom) et de l'armée kenyane, le chaos orchestré par les milices d'Al-Shabaab alliées d'Al Qaeda reste problématique. Son tracé va profondément perturber des circuits économiques anciens qui restent très dynamiques : en particulier tout le marché de la viande des clans somali qui profitent d'une économie de guerre. En outre, les provinces du North Eastern, d'Eastern et du nord de la Rift Valley en particulier, que doit traverser le corridor, ont été longtemps abandonnées par le pouvoir central, et sont source d'attaques intercommunautaires. Les centaines de milliers de réfugiés des pays voisins ont favorisé une prolifération d'armes modernes et bouleversé les équilibres de pouvoir au sein de ces sociétés pastorales. On ne compte plus les accrochages très violents entre ces bandes fortement armées et les forces de sécurité, faisant régulièrement des dizaines de morts, notamment à la frontière somalienne ${ }^{45}$. Les achats de terres à des fins spéculatives dans la région de Lamu et le long du corridor, d'une certaine élite kenyane, risquent d'aggraver les tensions. D'un point de vue environnemental, le corridor Lappset se heurte à l'opposition des communautés locales, peu consultées, dont celle des 25000 pêcheurs qui dépendent des ressources halieutiques de la baie. La pêche représente la principale source de revenus pour $70 \%$ d'une population peu instruite et donc peu employable dans de nouvelles activités ${ }^{46}$. Ces communautés craignent aussi que l'activité liée au développement du port ne menace l'héritage culturel unique de l'île de Lamu.

Enfin concernant le financement, il ne faut pas trop se laisser influencer par les déclarations d'intentions des pays membres. D'ailleurs le Kenya, hostile à un partage du pouvoir dans ce domaine, a décidé de prendre en charge l'essentiel du lourd financement de ces programmes ${ }^{47}$, hormis une partie de l'oléoduc endossé par le Sud-Soudan et une portion des chemins de fer du Lappset par l'Éthiopie. Alors que ces corridors entrent véritablement dans la stratégie régionale de l'EAC, les partenaires sans littoral interviennent peu dans le financement et n'ont pas, dès lors, de pouvoir de décisions. Comme de nombreux autres projets kenyans, ce montage financier s'appuie, en majorité sur des prêts accordés par la Chine, qui en contrepartie, bénéficie de crédit à l'exportation pour ses propres entreprises ${ }^{48}$. Mais la progression de la dette vis-à-vis de «l'empire du milieu » tend à fragiliser les autres types de financement comme le partenariat public privé (PPP) ou même les emprunts obligataires. Ce comportement de « franc-tireur » du Kenya peut avoir un double effet négatif. D'une part, en raison de cette fragilité financière, les travaux risquent de durer. Ces retards prévisibles ont d'ailleurs amené le Sud-Soudan à s'intéresser au port de Djibouti pour l'exportation de

45 Mérino, M. «Le Kenya : une locomotive régionale en panne », Géostratégiques, nº 25, octobre 2009.

46 Coloma, T. «En attendant le port qui doit sauver le Kenya », Le Monde Diplomatique, avril 2013.

47 Le coût de ces programmes s'élève à 327 millions SD pour le second terminal, 2,6 milliards USD pour la voie ferroviaire et 24,5 milliards USD pour le Lappset.

48 Ce partenariat plus pragmatique séduit les élites kenyanes qui souhaitent prendre leur autonomie vis-à-vis de l'occident et des organisations internationales. 
ses produits pétroliers ${ }^{49}$. Ce port, d'où transitent $70 \%$ des marchandises éthiopiennes, a pour objectif de concurrencer le Lapsset ${ }^{50}$. D'autre part, en gardant la mainmise sur les équipements, le Kenya peut continuer à imposer de manière aléatoire de nouvelles taxes que devront supporter les usagers de ces corridors ${ }^{51}$. Les pays enclavés, Ouganda en tête, se plaignent de cette dépendance et espèrent voir percer une réelle alternative.

\subsection{La voie tanzanienne pour compenser le pouvoir kenyan}

Ce tronçon est considéré comme une alternative pour contrebalancer le pouvoir des autorités de Mombasa et du Kenya en général, d'autant plus que le bassin du lac est une zone de convergence pour la plupart des routes transnationales de l'EAC. La modernisation et le prolongement des corridors tanzaniens auraient la propriété de non seulement favoriser la diversité des exportations des pays de l'hinterland comme le Rwanda et le Burundi, voire la province du Katanga mais également d'offrir un véritable débouché aux pays de la SADC dans le cadre du projet de zone libre échange SADC-EAC-COMESA ${ }^{52}$.

Cette rivalité, avec son homologue du nord, a amené la Tanzanie à appliquer les mêmes recettes en termes de financement ou de choix d'infrastructures pour devenir le $h u b$ portuaire de toute la côte orientale de l'Afrique. Ainsi, avec l'aide de la banque chinoise Exim, l'implantation d'un second terminal au port de Dar es Salaam doit élever les capacités annuelles à 600000 containers $^{53}$. Un projet d'extension de la ligne ferroviaire vers le Rwanda et le Burundi doit commencer, en 2014, en passant par les tronçons IsakaKigali et Keza-Msongati. La Tanzanie et l'Ouganda, de leur côté, s'efforcent d'accélérer la construction de la ligne ferroviaire entre les ports de Tanga et de Dar es Salaam vers Kampala via Malaka ${ }^{54}$. Mais suivant le modèle du "Lappset », c'est surtout le projet de construction d'un port flambant neuf à Bagamoyo destiné à devenir le plus important d'Afrique, avec une capacité de 20 millions de conteneurs traités annuellement, qui

49 Les travaux sur le port de Lamu devraient prendre environ 4 ans dans le meilleur des cas selon les autorités portuaires du Kenya (KPA). Or le Soudan du Sud ne peut attendre si longtemps. Le pétrole fournit $98 \%$ de ses revenus et Lamu n'est pas le seul débouché possible. Petro'Trans, entreprise basée à Hong Kong s'efforce de développer en Ethiopie et en Somaliland les installations portuaires, de gaz et de pétrole afin de les connecter par la suite au Sud Soudan. Africa Research Bulletin, février 2012.

50 En 2011, le port de Djibouti a traité 705000 containers. L'objectif affiché pour 2013 est de 1 million de containers. "Juba and Addis: Why the coffee smells so strong in the North », The East African, 29 juin/5 juillet 2013.

51 Les autorités portuaires du Kenya (KPA) ont d'ailleurs récemment émis une taxe de 300 dollars pour chaque container en direction de l'Ouganda. On parle d'une taxe de 5 Kenyan Shilling par litre d'essence, une taxe verte, une autre sur les cargaisons traitées par le port de Mombasa, et un surcoût de plus de 10 millions USD aux nouveaux opérateurs ainsi qu'à RVR. « Congestion at Mombasa port slows down trade in EAC bloc », The East African, décembre 2012.

52 Common Market for Eastern and Southern Africa.

53 Intéressés par la découverte de gaz dans le Sud-Ouest de la Tanzanie les chinois sont d'autant plus motivés pour moderniser le corridor sud.

54 Africa Research Bulletin, février 2012. 
risque de modifier radicalement le rapport de force ${ }^{55}$. Une connexion au réseau ferroviaire tanzanien et à la ligne Tanzanie-Zambie (TAZARA) est par la suite prévue afin de desservir l'ensemble de la sous-région, du Soudan au Botswana, en passant par la RDC. Les routes continueront d'être financées là où elles sont essentielles pour combler les chaînons manquants ou soutenir la prochaine phase d'une opération en cours. Les résultats attendus, pour le corridor central, sont une baisse de 32 dollars par tonne transportée et une hausse de $25 \%$ du commerce ${ }^{56}$.

Mais ces importants investissements proviennent pour l'essentiel d'un seul pays, à savoir la Chine. Les pays d'Afrique orientale se trouvent de ce fait dans une situation de dépendance à l'égard de ce partenaire privilégié. Un retournement de conjoncture, dans l'empire du milieu, risquerait de réduire considérablement ses investissements à l'étranger dont souffriraient en premier lieu les pays d'Afrique orientale. Par ailleurs, la compétitivité des corridors tanzaniens exige, une restructuration du système logistique. Or, les processus de privatisations des compagnies ferroviaires publiques, Tanzania Railways Corporation (TRC) et TAZARA, sont à l'arrêt. Et celles des ports de Mwanza et de Port Bell, primordial pour un trafic maritime efficace sur le lac Victoria, ne sont même pas à l'étude. Enfin, l'absence de la Tanzanie, ainsi que celle du Burundi, au sommet informel d'Entebbe de juin 2013, laisse apparaître un nouveau positionnement géopolitique de l'Ouganda et surtout du Rwanda avec le Kenya. Cette consolidation du corridor nord risque de porter atteinte à la viabilité des projets tanzaniens, notamment celui de Bagamoyo lequel devrait dépendre alors davantage du dynamisme des échanges avec la SADC.

\subsection{Un impératif d'approfondir l'EAC}

L'un des objectifs de « Vision 2030 » est clairement de faire accéder le Kenya au statut de pays à revenu intermédiaire. Pour atteindre cette cible le processus de croissance ne peut se restreindre à la seule zone du « Kenya utile» des hauts plateaux. D'ailleurs les régions arides peuvent profiter des corridors, dont le lapsset ${ }^{57}$ et ce grâce aux investissements relatifs aux transports même si ces derniers sont destinés avant tout aux pays voisins ${ }^{58}$. Cependant, il semblerait plus efficient de développer d'abord les zones portuaires à partir desquels des forces centrifuges pourraient apparaitre.

En effet, selon Hirschman ${ }^{59}$, lorsque des activités directement productives (ADP) se développent, l'amélioration des infrastructures économiques et sociales (IES) devient un véritable soutien logistique à leur accroissement. Le Lapsset dont la mise en place doit

\footnotetext{
55 Suite à la visite du président Xi Jinping en mars 2013, la Chine s'engage à investir 10 milliards UDS pour la construction du port de Bagamoyo attendu pour 2017, Jeuneafrique.com: Tanzanie: Le plus grand port d'Afrique attendu pour 2017.

56 Trade Facilitation in the East African Community..., op. cit.

57 Selon Hervé Maupeu, les tendances de croissance insufflées par «Vison 2030 » se feront dans les périphéries, ce qui est contraire à «l'ADN du Kenya indépendant».

58 Malgré les discours de M. Kibaki sur l’injustice historique subie par les zones périphériques, le projet

LAPSSET est d'abord conçu comme un système coordonné de transport destiné aux pays enclavés.

59 Hirschman, O. Stratégies de développement économiques, Paris, Editions ouvrières, 1964.
} 
permettre au Sud-Soudan d'exporter son pétrole, l'illustre parfaitement. En revanche, le seul développement des IES ne suffira pas à créer des ADP dans les zones arides situées au nord du Kenya. D'ailleurs, la présence dudit corridor peut certes faciliter l'accès, et donc l'activité agricole, de ces zones du North Eastern mais aura du mal à attirer de nouvelles ADP.

Pourtant, certaines études ${ }^{60}$ tendraient à démontrer que si l'infrastructure progresse plus rapidement que les activités productives, cela stimulerait la croissance. Certes mais sous certaines conditions : le développement des IES peut effectivement être favorable au développement d'ADP si les territoires concernés disposent d'atouts pour permettre de développer des économies d'agglomération. Les expériences étrangères, notamment en Asie, nous montrent que ce sont notamment les «zones d'intégration côtières » qui améliorent en partie la compétitivité des provinces chinoises, ou des cités États comme Hong Kong. Etant davantage proches des marchés mondiaux, elles réduisent fortement les coûts de transport tant pour les intrants importés que pour leurs produits industriels ${ }^{61}$. L'optimisation des équipements, les échanges d'information et les économies d'échelle favorisent le climat des affaires et sont facteur de rendements croissants. L'attrait d'investisseurs, notamment étrangers qui diffusent leurs technologies, ont permis l'éclosion de « clusters » performants au niveau mondial.

La transformation des installations portuaires de l'Océan indien, la modernisation des corridors et la création, en 2012 par le Kenya, de zones économiques spéciales $(\mathrm{SEZ})^{62}$ autour de Lamu et de Mombasa pourraient certainement contribuer à booster la compétitivité des entreprises régionales en quête de marchés mondiaux. "Les régions côtières redeviennent le pivot de l'espace économique libéralisé ", selon François Gripouloux $^{63}$. Mais l'un des facteurs d'attractivité, à savoir la densité de population, notamment pour la main d'œuvre qualifiée se situe surtout autour de Nairobi et du bassin du lac Victoria, à plus de 500 kilomètres du littoral. Il serait donc judicieux d'encourager la mobilité du facteur travail qualifié vers ces zones côtières qui en sont dépourvues, plutôt qu'espérer voir se développer des activités dans les zones périphériques. L'application sur le terrain du Marché Commun d'Afrique de l'Est, officiel depuis 2010, serait un excellent moyen d'y parvenir.

60 Analyse empirique réalisée par J. Weiss, pour le compte de la BAD, concernant 31 pays d'Afrique dont le Kenya et la Tanzanie, pour évaluer l'impact de la longueur des routes et de la puissance installée des centrales électriques par habitant sur le PIB. Voir Rapport sur le développement en Afrique : le développement des infrastructures en Afrique, Banque Africaine de Développement : BAD, 1999.

61 World Bank, East African Community.., op.cit.

62 La loi sur les zones économiques spéciales est entrée en vigueur en novembre 2012. Elles concernent au Kenya les régions de Mombasa et Lamu sur la côte, Kisumu et le lac Victoria. Bénéficiant d'une législation et d'une fiscalité avantageuse, de terrains prêts à bâtir et d'infrastructures modernes, ces zones sont conçues pour attirer les activités industrielles et les investisseurs internationaux.

63 Coloma, T. « En attendant le port...», op.cit. 
En tout état de cause, un renforcement progressif du rôle des institutions régionales semble être indispensable pour améliorer l'application de la législation en vigueur. Leur confier un pouvoir de coercition plus important pourrait être bénéfique aux acteurs, ces organisations doivent cependant principalement œuvrer pour faciliter et favoriser le commerce. L'intégration par le haut ne doit pas desservir une intégration par le bas. Ainsi, en étant davantage présentes dans la surveillance et la gestion des infrastructures transfrontalières, elles pourraient accélérer la suppression des barrières tarifaires. Celle-ci a pourtant été votée en 2012 par les ministres de l'EAC mais sans sanctions appropriées ${ }^{64}$. Il est vrai, cette mesure touche un point extrêmement sensible puisqu'elle implique une altération des fonctions régaliennes des états, et en particulier du Kenya. Ce refus des partenaires de voir disparaitre une partie de leur souveraineté explique, en partie, les difficultés de l'EAC d'avancer de manière efficiente.

Par ailleurs en dotant l'institution régionale d'une réelle autorité sur la conception du système d'infrastructure régionale, elle pourrait davantage servir l'intérêt collectif. Une participation de l'hinterland au financement des corridors, en échange d'un pouvoir de gestion et de police sur le fonctionnement des ports et des routes, permettrait d'optimiser l'efficacité de la structure logistique. Les producteurs de produits agricoles ou d'extraction, étant par nature immobiles, auront la possibilité d'accéder plus facilement aux marchés mondiaux. De telles décisions inciteraient l'EAC à tendre vers une union fiscale. L'application d'une taxe unique des droits de douane à l'arrivée au port serait ainsi un moyen efficace de simplifier et de fluidifier les transits sur les corridors ${ }^{65}$.

Enfin, pour permettre une intégration politique soutenable, il semble urgent de prendre des mesures compensatrices pour les régions les plus démunies. S'il est difficile, voire inefficient, d'imposer l'implantation d'activités, un effort dans la réduction des disparités d'éducation serait favorable à une hausse générale de la productivité de la main d'œuvre. Dans ce cadre, les bailleurs de fonds internationaux pourraient agir en accordant des prêts aux zones les moins avancées tant dans les infrastructures physiques que dans les services sociaux ${ }^{66}$.

\footnotetext{
64 La loi d'harmonisation des standards de transport routier, votée en 2012, a pour finalité de faciliter les passages de frontière et lutter contre les barrières non tarifaires. «EAC Ministers Approve Roads Bill », The Daily Nation, 20 February 2012.

65 Lors du sommet informel d'Entebbe, l'Ouganda, le Kenya et le Rwanda ont discuté de l'éventualité de mettre en place des zones douanières uniques : le port de Mombasa au Kenya ; Mpondwe en Ouganda et Gisenyi au Rwanda. Voir «Bilateral and Tripartite Deals : Presidents Agree to Fast Track Political Federation », The East African, 29 juin/5 juillet 2013.

66 World Bank, East African Community..., op.cit.
} 


\section{Bibliographie indicative}

Akanni-Honvo, A. \& Léon, A. « La croissance endogène régionalisée », Revue Tiers Monde, $\mathrm{n}^{\circ} 155$, juillet-septembre, 1998.

Alix, Y. \& Pelletier, J.F. « Territoires enclavées et opportunités de marché: analyse des performances logistiques des corridors de transport en Afrique subsaharienne », Organisations et territoires, 2011.

Behar, A. \& Venables, A. Transport Costs and International Trade, Oxford Department of Economics Discussion Paper 488, Oxford, University of Oxford, 2010.

Blanc, M.O. Stratégie des acteurs, dynamiques urbaines et structuration des territoires. Sous projet Afrique Australe, Paris, PRUD, projet n³7, janvier 2004.

Calas, B. « Le système logistico-urbain est africain », Mambo!, IFRA, 2000.

Cirera, X. « Regional Integration in EAC Countries: Lessons and the Way forward », Institute of Development Studies, 3 January 2013.

Gonzalez J, Cirera, X. «A Review on the Impact of Transport Costs on Trade Flows », Institute of Development Studies, Final Draft, 28 November 2012.

Hirschman, O. Stratégies de développement économiques, Paris, Editions ouvrières, 1964.

Krugman, P. « Increasing Returns and Economic Geography », Journal of Political Economy, n99, Vol. 31, 1991, pp. 483-499.

Médard, C. « Quelques clés pour démêler la crise kenyane : spoliation, autochtonie, et privatisation foncière », Les élections générales de 2007 au Kenya, Nairobi/Paris, IFRA/ Karthala, 2008.

Mérino, M. « Le Kenya : une locomotive régionale en panne » Géostratégiques, $\mathrm{n}^{\circ} 25$, octobre 2009.

Porhel, R., «Les conséquences économiques de la crise politique », Les élections générales de 2007 au Kenya, Nairobi/Paris, IFRA/Karthala, 2008.

Shepherd, B. « Geographical Diversitification of Developing Country Exports », World Development 38.9: 1217-1228, 2010.

Society for International development, The State of East Africa 2012, Deepening Integration, Intensifying Challenges. Nairobi, SID, 2012.

US International Trade Commission. Trade Facilitation in the East African Community : Recent Developments and potential Benefits. Publication n4335. July 2012.

World Bank. Transport prices and costs in Africa, BIRD, 2009. 
Observatoire des Grands Lacs en Afrique

World Bank. "The Africa Competitiveness". World Economic Forum Report, 2013.

World Bank. East African Community. Reshaping Economic Geography of East Africa:

From Regional to Global Integration, Report n65699-AFR, June 2012.

\section{Presses \& Sites Internet}

Africa Research Bulletin, January, February, July 2012.

Le Monde Diplomatique, avril 2013: http://www.monde-diplomatique.fr/2013/04/ COLOMA/48968

The Daily Nation

The East African

Jeune Afrique : Jeuneafrique.com

Tanzanie : le plus grand port d'Afrique attendu pour 2017. 
Annexe 1 : Carte de l'EAC

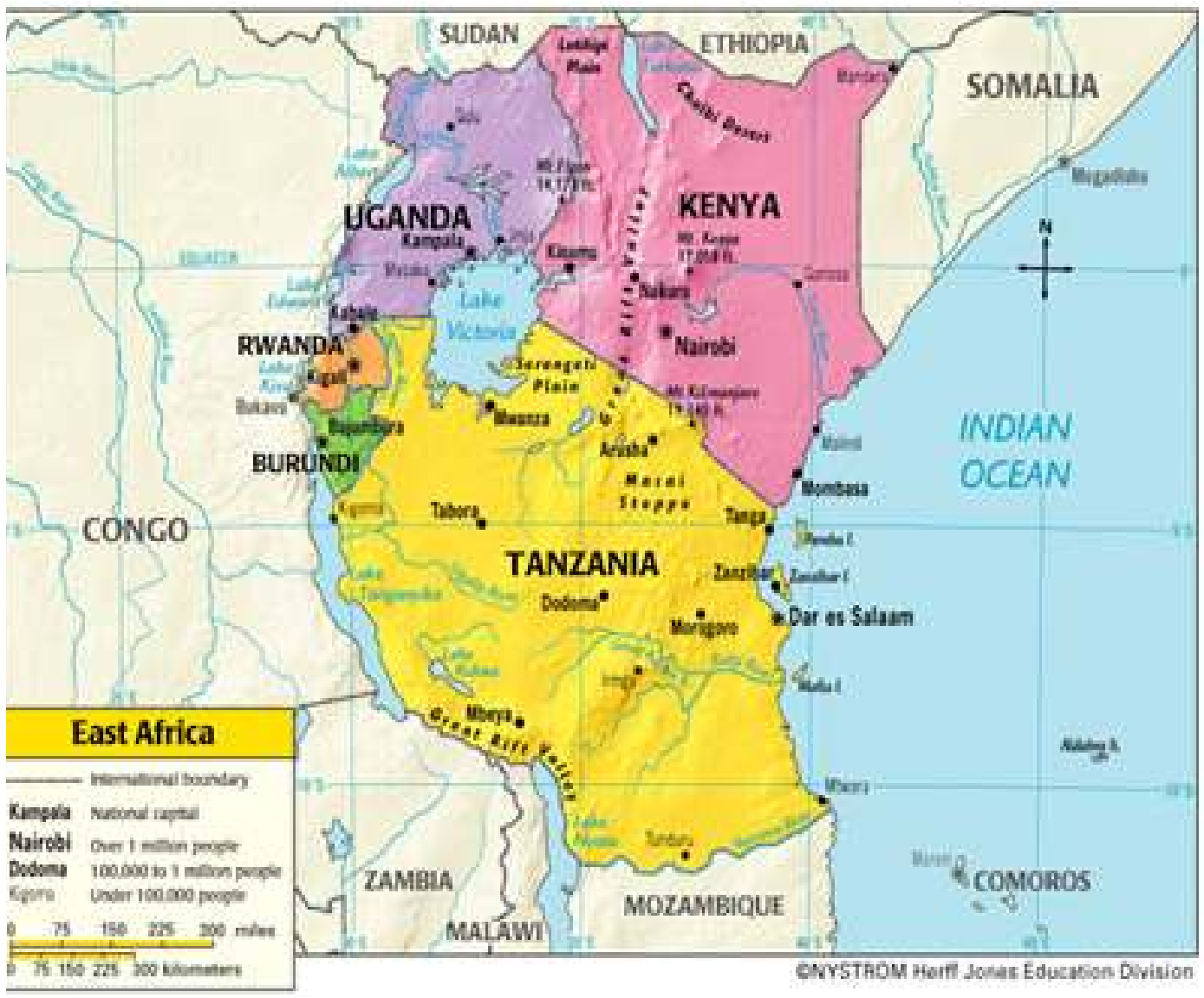


Annexe 2 : Carte des corridors en Afrique de l'Est

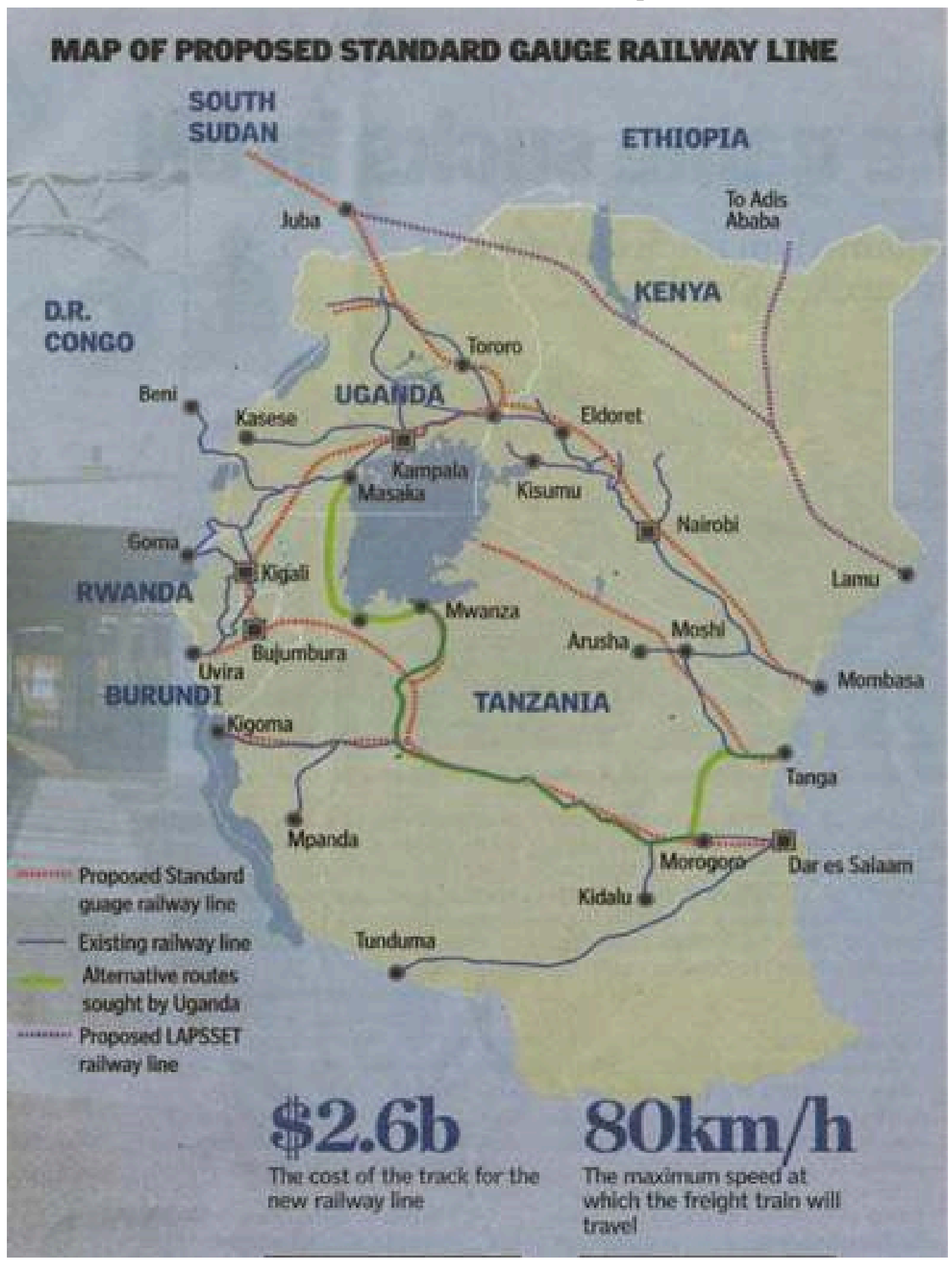

